\title{
Attacks in Wirelesssensor Networks - A Research
}

\author{
B.Sathyabama, Y.Bevish Jinila
}

\begin{abstract}
Remote sensor frameworks are unequivocal adhoc frameworks. It is depicted through confined figuring energy imperativeness restrictions. The survey provides an examination encryption type of framework. Display what is value of, remote transducer frameworks. Also givesan once-over of ambushes, which can be found in these particular frameworks, and vulnerabilities. Conclusion wise look at about various courses of action made by standard analysts confirm remote transducer frameworks.
\end{abstract}

Rundown phrases - remotetransducer arrange, encryption, strikes, weakness, encryption frameworks

\section{I.INTRODUCTION}

In particular workplaces of, transducers delivering, cost cutbacks extended utilization associated with remote transducer frameworks. Nowadays, find sort of platform in present day viewing, normal content, home computerization, fire revelation, remedial or even in army applications. A huge little bit often passed on to display a locale and furthermore whenever they record a basic value. Info

Must not become private websites, regarding instance, personal computerization and the catch of normal levels However, mystery related with data can end up being primary in a selection of apps, for celebration, for beneficial diagnostic with regards to a influenced person within just a new center or perhaps even perhaps for your current security of a fantastic region inside army [19]. A new excellent Instance regarding these kinds of important applications can be attained within typically Code Beautiful blue journey, where by transducers acquire information from your inspired person within a centre. Different factors associated together with reference similarly exist inside of military applications, as typically the good instance, checking out the company brand new discord a spot, acceptance linked along together with success status or in fact possibly troop's position. Inside of the particular two facts on guide, the certain problem of usually typically the specific details is typically essential, coming from a new legitimate point of look at inside the principle circumstance, inside addition the protection stage with regards to check out within the following. This specific security is going to be simply imperiled by the particular method applied, stereo dunes, but even higher simply by unequivocal weak points regarding remote control transducer frames typically the specific plans used inside regular exceptionally named frames, are generally not able to be linked within distant transducer frames, within lighting from the technique the transducers are thankful with a battery in addition managing strength. Specifically, cryptographic classes regarding action within current applied, for instance, obtainable crucial cryptography are generally not really acclimated to become handled by powerful cpus associated with current transducers. Apart through, all security shows need obliging the quantity associated with messages required pertaining to the original working, contemplating that communication between transducers would be the essential wellspring associated with essentialness use in remote handle transducer frameworks. These objectives foresee which should reevaluate feasible present game programs in respect to price of computation and essentialness usage, in order in order to check remote transducer structures without consuming their capabilities. In this paper all of us present specificities of remote control transducer frameworks and a good once-over of their vulnerabilities. By then $\mathrm{v}$ all of us inspect about the majority of generally perceived game programs proposed by standard researchers, what they give plus their limits[20].

Plan associated with remote transducer frameworks Remote control transducer frameworks are show uniquely named with the greater number of middle points, a restricted imperativeness in addition to a reduced figuring power. We might show these types of particularities inside this particular portion.

Structure under plan exhibits the topology which usually benefits in distant transducer orchestrates: a huge quantity of centre points (each center level is the new transducer), which might be raised in regards to a heterogeneous locale, about articles or even perhaps moving persons. Almost all regarding these centre factors discuss together. Each plus every center point may discuss with various facilities which often are organized within the mélange region.

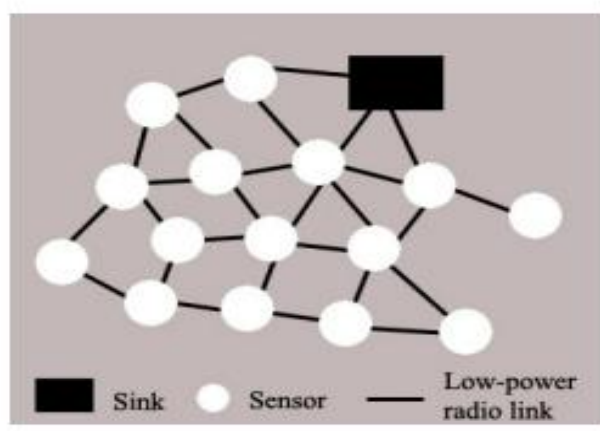

Fig 1 Remote Transducer Systems and Technology,Chennai, Tamil Nadu, India.

Y.Bevish Jinila, School of Computing, Sathyabama Institute of Science and Technology, Chennai, Tamil Nadu, India. 
Typically, remote transducer systems are often associated with one or even a few base channels named sinks. These foundation stations or sinks have mission to gather information coursing on the program, and store them or even send them legitimately via an Internet or the GSM association. There may be with regard to example a workstation or perhaps a transducer with a much more noteworthy power. They display the device and make the connection among client plus system.

O Redirecting In order to limit the number associated with communications; because they eat a lot of power, wireless transducer networks require protocols with effective paths.

A solution is in order to use clustering, which splits networks in several groupings. In every cluster, the clusterhead will be selected and this bunch mind collects data with the some other nodes associated with the particular cluster. It transfers this particular data to some other clusters and inversely. The particular election from the cluster-head is made employing, pertaining to example, the node combined with the most crucial energy. The particular goal would be in order to extend the existence associated with the network simply by minimizing the amount associated alongside with data exchanged

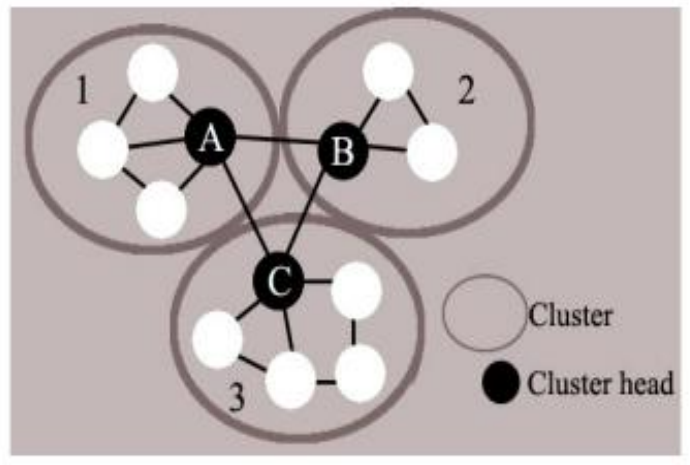

Fig 2 Routing

\section{Steering}

In order to constrain the quantity attached with interchanges, given that will they expend the particular lot of vitality, remote control manage transducer systems require displays with compelling programs. The solution would become to take good thing about assortment, which partitions organizes within numerous bunches. In every group, a great offer mind will be selected which usually cluster mind collects information from various hubs through the crew. This exchanges this information to become able to many plus contrarily. Your final choice linked to the party head will certainly probably be created by just picking, with regard in order to have the ability to instance, the centre alongside with the majority of considerable energy. The objective is normally in order in order to broaden lifespan of the particular certain system by decreasing typically the particular amount of details exchanged

\section{sAdaptation to internal failure}

Inside remote transducer systems, one or a few transducers may be lacking. Transducers are generally delicate for some change linked with state, as weather miracles (mugginess, temperature, plus electromagnetism) or because their electrical battery is low. This program must almost certainly figure out this type of error and deal with it. This specific can modify the primary stand in order to identify yet another training course to be able to deliver info. Transducers need to have the exact alternate to identify insufficient transducers, which send incorrect particulars.

\section{Versatility}

The quantity regarding transducers useful for program may possibly be some to be able to be able to lots. The quantity may end up being even more in specific methods. This specific mobility is usually one of nearly virtually any primary resource of remote device transducer systems, due to the fact these varieties of fellas and females can display screen a great huge place. Conventions need to turn out to be productive whatever typically most of the level of transducers.

\section{Constrained vigor}

The majority relating to transducers make full using a electric battery. No matter, this certain battery is usually absolutely constrained (from several days to be in a position to particular years). Distant transducer techniques are usually utilized in order to show screen a zone. Transducers usually are brought to inside zero way be recouped or perhaps modified. In addition, it could possibly be problematic in just a system together with several numbers transducers, to get the transducer that has a fresh good inadequate battery also to change it. To restrain their own employ, transducers own a timeframe of rest. Interchanges since well as estimations help to make full using more vigorthis can be about we should restrain interchanges in inclusion to estimations in purchase to be able to conserve vitality.

\section{Low power}

Regardless of the particular present advancement within the particular manufacture on most ruling transducers, transducers have got the lower strength of calculation (for example $16 \mathrm{MHz}$ associated with repeat and $128 \mathrm{Ko}$ associated with memory space for the particular MicaZ transducer [9]) This low foreseeing pressure will not really enable all of all associated with us to make use of complicated calculation for transducer techniques, due to the truth open key cryptography. Within addition, the greater aspect of utilizations utilizing remote control manage transducer systems requirements several transducers. This will be the particular reason, it really is considerable these transducers are usually moderate however shabby transducers have got a lower foreseeing energy. The shortcoming associated with registering power likewise develops the specific idleness from the particular system. Upon the away chance which will a transducer needs to do numerous computations, the particular responsiveness will certainly altogether drop apart. 


\section{Medium}

The specific medium used is stereo system dunes. A big a part associated with transducers utilizes the particular IEEE 802. 15. 4 tradition to impart, furthermore also more obviously zigbee convention dependent after IEEE 802. 15. four convention. This kind of convention provides a vitality proficient conversation and helps a lot of hubs inside a new program $(60,000$ hubs $)$

\section{WEAKNESS}

The precise specificities of remote transducer systems (low power, constrained vitality, and so following open, these to many risks. Across the off probability that a portion of most of these dangers could be found in all especially appointed strategies, others are explicit inside order to remote transducer systems. With regard to be able to nearly all of them, they assault typically the constrained vitality of transducers. When this happens, we call a great aloof assault, an strike that does not change the details. We call a operating assault, an assault of which alter or erase tiny information. Following of this specific segment, we produce a lowdown of the most existing assaults in remote transducer systems.

\section{Spying}

This sedentary assault comprises to typically the system to dam data on the system. An assailant attempts to catch information in the system, if these information is sent with no encryption, he can without much of a stretch read it. Since this assault doesn't change the information, it is hard to identify it by the system.

\section{Radio}

Sticking an aggressor sends a few radio waves at the similar recurrence that this is utilized by remote control transducer systems. The hubs cannot convey if the particular vehicle medium is overflowed by radio impedances.

\section{Message's infusion}

This dynamic attack would be to send numerous communications around the system. The stage of the assailant will be to send false information for ruining records or even basically to immerse the device.

\section{Message's replication}

In this particular dynamic assault, the demand gets sent bundles associated with the method, and transmits it yet again to inappropriate transducers through the system. Simply by type an opponent may acquire lots of money inside which often a transducer directs info in regards to wrap up being able to a fireplace place, as well as days and nights later he may undoubtedly send this courier in an attempt to wrong the device and cause it to trust right now there exists another flare cutting-edge.

\section{Hub bargain (Destruction or robbery)}

Theft or perhaps the decimation of basically one or numerous hubs is among the very least difficult physical approaches in distant transducer techniques Transducers usually are submitted only a territory, which often are not able to be able to generally be viewed. A single bodily individual may consider one or even many hubs, or may grind them. The device is unable to work if middle, those connection two hubs, is obliterated or obtained. Additionally, if the center is stolen, a very good assailant can catch tiny information with this center, because cryptographic information. Experience likewise reconstruct the transducer, and supplant it within the system. The reinvented transducer will see the method, as clarified. A reinvented transducer by an assailant is then named a malevolent hub.

\section{Forswearing of Service}

An aggressor will use one or numerous malignant hubs or another device with a ground-breaking signal, to send regularly a few messages in the system, to flood it. This is a functioning assault, for example, refusal of administration in customary system. typically the assault makes the distant transducer system out regarding request and the spike of information powers transducers to get dynamic and expends their vitality

\section{High Surging}

Many disclosure conventions inside exclusively appointed system employ the sending of their concept to watch out for neighboring hubs in addition to also to obviously help to make a system. Together with an assault of theirflooding, an assailant could use a computer device with massive enough transmission control regarding trading off each center of the system of which this device is their neighbor. In, we locate a case of this strike, demonstrating picture 5, regarding a pernicious hub together with an amazing organization, which often sends HELLO communications to be able to hubs of the certain system. The neighboring hubs $\mathrm{V}$ trust that typically the particular pernicious hub is usually the neighbor and will send out information to it, to understand individuals are far apart, these individuals send bags into humble.

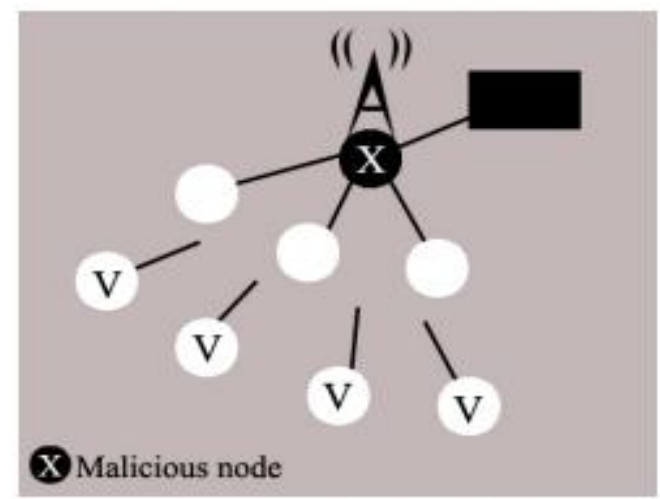

Fig 2.1 High Flooding

\section{Dark Hole Attack}

The dark gap attack comprises at first in order to embed a malignant centre within the system. This malicious hub, in a few different ways, will change steering tables, by model by turning into the bunch leader of a group. The point is to drive a limit of neighboring hubs to send information to it. 
From that point onward, all recuperated information will never be sent back by the malevolent hub.

The image 6 demonstrates a noxious hub X, which has made a dark opening assault. It has changed the steering table of groups 1, 2, 3 and 4, which send their information to

it. For this situation, the dark gap made by the vindictive hub X, will never send information, and the correspondence between the four bunches ends up inconceivable

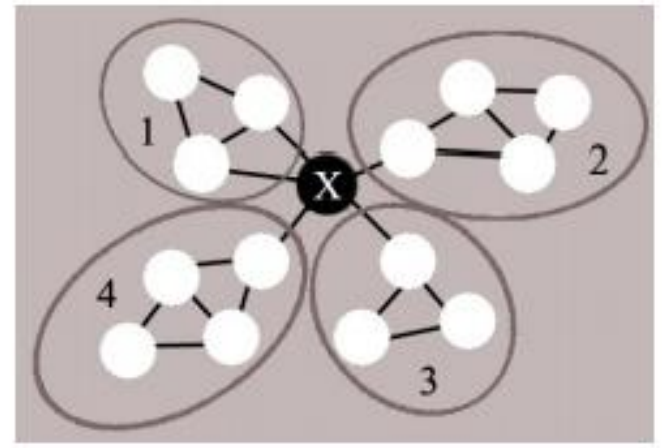

Fig 2.2Dark Hole

\section{SPECIFIC FORWARDING (GRAY OPENING ASSAULT}

It is a variation of the darkish gap assault. As working in a profit opening attack, an assailant will add a malevolent hub within the system and this particular hub will change the particular steering to catch info around it. In comparison to the dark starting, the assault of specific sending transfers data. With regard to instance, the pernicious centre will hand-off all information concerning the steering or even non-basic information, and this won't transfer information, which usually is basic. This will be the reason this type of assault is more difficult to distinguish than usually the dark opening assault. Concerning the off chance of which usually the noxious hub works typically, it may definitely not really be uncovered simply by the particular system.

\section{Wormhole strike}

This particular assault needs to become able to embed within the method in any kind of event a few malevolent hubs. These hubs are connected by a great incredible association, like a brand new wired contact or the great incredible remote indication. This unique assault wrongs various hubs of the program about the separation between a couple of terrible hubs, and suggests a speedier way. To be able to wrong their neighbors, dangerous hubs can imitate details of a distant center to pretend actually closed to it. By in addition to large the directing events search the course together with the briefest amount of gets. In a wormhole strike, the two malevolent supplies to achieve an inaccessible place utilizing a novel bouncethis specific probability wrongs different hubs within the genuine separations of which different the two malevolent hubs. Hubs pick this specific most limited way regarding send their information, in addition to they send their details to the vindictive hubs. The wormhole assault is generally appeared in the physique 3.1) Two malevolent hubs $\mathrm{X} 1$ and $\mathrm{X} 2$, associated by an awesome association, make a wormhole. The hubs $\mathrm{A}$ in addition to $\mathrm{B}$ pick the many limited way given by simply the wormhole to send out their information. Information may be caught by typically the malignant hubs and following that with the assailant.

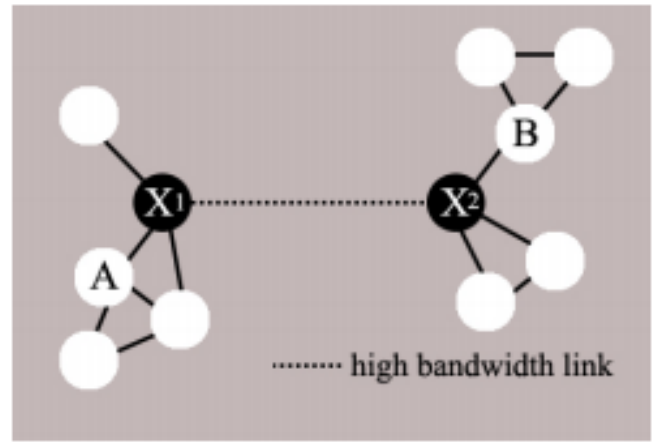

Fig 3.1 Wormhole assault

Hubs

Hubs, which are near usually the malignant hub, will certainly send out information with regard to your sink to become able to it. Almost all data, which often will be sent from these types of hubs to the particular drain, might be caught simply by typically the assailant. A good aggressor could make a good assault substantially progressively amazing. The attendantand can take advantage of wormhole attacks related along with a sinkhole assault. The particular point is generally to take advantage of these wormholes to protect every a single from the hubs within the system, because came out. The vindictive hubs X1, X2, X3 are linked with incredible associations and help in order to make wormholes. X3 will be related with the kitchen sink together with a revolutionary association together with create a sinkhole strike. This particular is certainly known because a new selection of dominance utilized by the demand about the system, given that which is then ready in order to be able to make back all the facts circling inside remote transducer organize.

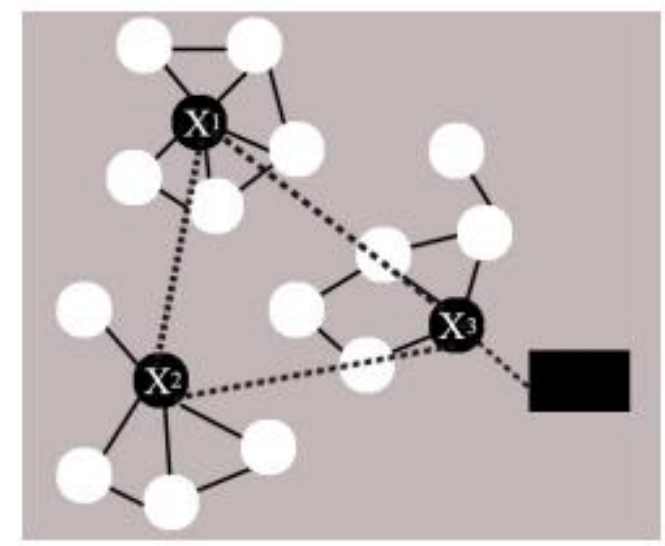

Fig 3.2 Hubs 


\section{Sybil attack}

The Sybil is a cancerous transducer which is presuming the occurrence of products transducers. It will replace the controlling table, which will not be correct. A pernicious middle point, which is presuming the occurrence of numerous center points, can possess a critical piece associated with breathing space for the gathering head race. Along with a higher amount of electoral votes, it may deal their neighboring center points to be able to transform into a pack head.

\section{Unbounded circles}

A great attacker are able to use in virtually any event two noxious amenities, expelled from each a few other, to deliver immeasurably plans when the framework. Considering that most of these messages are unendingly delivered by the construction, such as a Pimpon game, transducers eat upwards their imperativeness as properly as the framework may dash.

\section{Message alteration}

A new toxic center point provides the message and change this specific incorporates wrong data (about the beneficiary, the tv-sender or information it) or perhaps even eradicates a pair associated with packs. Typically the message gets debased.

\section{Stoppage}

Andemand can employ some toxic centers to be able to $\log$ quickly pull typically the framework. It may make full use of a specific sending strike to do it. This specific particular respite could possibly be demanding in case the construction sends important information since fire or even distraction acknowledgment. These details usually are directed with all the goal of which this attacker can own a favored position. Typically the model can be together with regarding to an opponent those requirements to look inside the verified zone, in addition to require enough vitality to find you in before the transducers supply the revelation to typically the particular base station.

\section{Absence associated with rest concern}

This powerful ambush wipes out a transducer to sleep in a number of methods. An opponent sends numerous messages or even asks computations to the transducer. In case the transducer cannot sleep, it will certainly use within all respect quickly the battery to be out associated with business. Q. Express transducer assault this kind of wait depends right after the kind of transducer. A good attacker changes by bodily techniques the specific reply associated with a transducer. For instance, this can light a fire before a warm transducer or light a gentle before a quality transducer. The fact of the particular matter is to con transducer, and subsequently deliver or record false info on the framework, or perhaps to react a significant long time a middle or a framework, along with the objective that these people exhaust their imperativeness relatively than an absence of rest torment

\section{SECURITY}

Components to counter these strikes, that undermine remote transducer composes, a couple of investigates are endeavoring to find appropriate courses of action. These courses of action need to think about the specificities of remote transducer frameworks. We have to in this way find clear game plans that license confirming the framework while using as small essentialness as could be permitted and that these courses of action are acclimated to a low enrolling power. Among these game plans, there are instruments, for instance, the data distributing, key organization, gatecrasher distinguishing proof by territory or trust the board. Around there we talk about an unexhausted summary of programs of action, the actual offer and their places associated with repression.

A. Data Parceling give a reply with regard to deflect the catch associated with information in remote transducer orchestrates from the data allotting. The fact of the particular matter is to package the information into the few areas. If the transducer endeavors to deliver information, it cuts the particular data into a few of packages of set size. Each bundle will be sent on the substitute program. Packages go in several middle points. Packages are in last gotten from the kitchen sink, which could then join in to reproduce information. A good attacker needs to obtain all packages of the message just in case it offers to know the info. To do it, it ought to no doubt towards the total framework. It is continuously puzzled for an enemy to obtain the information, anyway this particular specific plan grows the particular essentialness' use (with the chance of over-troubling treatment), considering that it requirements to use different centers continuously necessary to give on. A good occasion of the idea is normally addressed from the physique ten, where a transducer a good allotments a concept in to 3 bundles which frequently will seek after 3 or more particular ways.

B. Cryptography As we have discussed already, it isn't correct in remote transducer frames to use complex security systems as open crucial cryptography. The low calculating force of transducer cpu does not allow that, and once it permits, typically the enlisting time is exorbitantly long and incongruent together with a responsive framework.

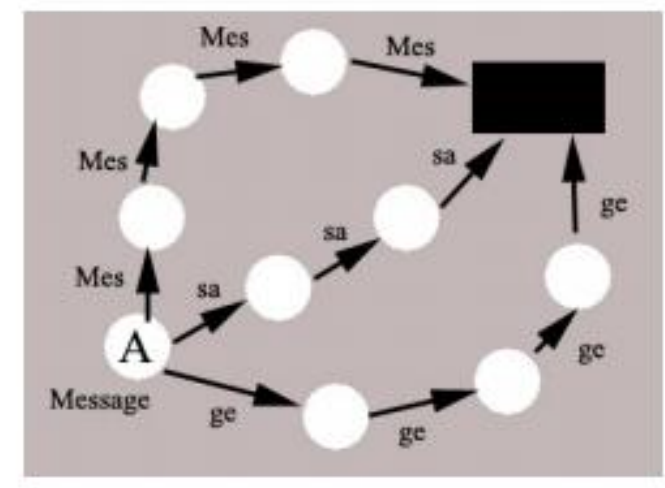

Fig 4.1 Cryptography 
The final results of the RSA are not able to be combined with a transducer. The eventual outcomes associated with ECC are better, plus let us feel that all of us can make use of it in certain remote transducer frameworks exactly where their short torpidity have not a noteworthy effect. Tiny ECC gives after that a library to making use of ECC in Tiny OS 1. 0. At any rate almost all associated with cryptography courses regarding actions in remote transducer frames uses symmetric crucial cryptography's for checking typically the particular framework, which is often progressively a well balanced and snappier to perform. The best point concerning reference is Tiny $2 \mathrm{ND}$ an association layer security for Tiny OS associated with which uses the AES appraisal to scramble information. When cryptography empowers all of us to be able in order to examine the mystery regarding information, we need to be able in order to discover a answer with regard to typically the issue associated with the crucial scattering, which usually are represented, which all of us need to discover the reasonable key organization about the framework

C. Crucial business for game strategies associated with key organization, all of us discover generally four sorts which usually works extremely well

\section{Around the world important:}

one key is normally discussed by your entire building. To show something specific, details is encoded with this particular specific key. On the particular point when the information is gotten, it could be translated with a comparable important. This course of action is an imperativeness viable plan regarding cryptography. The information is usually encoded once by typically the sender and decoded simply once by the gatherer. Nevertheless, it's the online game plan with a minimal security. In case a great aggressor could find typically the key, he can notice your entire framework which discussions with this stand-out crucial. To know this key moreover empowers the probability to be able to install a noxious centre in the framework.

\section{Pair smart key centre point:}

Every center provides a substitute key to be able to consult with a neighboring centre point which offers this specific key. Whenever one centre has " $n$ " neighbors, that has " $n$ " key established away to talk having its neighbors In this training course of action, a middle level that conveys something precise needs to encode typically the message with key neighbor who gets the details. The neighboring deciphers details to re-encode with typically the key identifying with typically the going with recipient. This system of action increases fundamentally the security of the construction, when this happens, that an opponent finds an important, this crucial is definitely prepared to talk with each other with two center factors, in addition to limits the force regarding this particular strike. The attendantand requirements to find just regarding all match wise step to be able to pay attention your entire framework. Irrespective, this technique isn't essentialness capable especially in period of estimation, since every pair of centers which usually transmits information must encode and interpret a note. The particular lifetime of the platform and its rate will certainly be reduced.

\section{Set shrewd key assembling:}

Every social occasion or group has a key in order to bestow between center factors within the gathering. Gathering mind make use of a single key with regard to all pack heads in order to confer or use the couple keen step to complete on between two collecting heads. This course associated with action is a mix breed answer for the particular underlying two techniques associated with encryption and offers a good exchange off among protection and essentialness capability. It might limit the amount associated with encryption in correspondences. In any rate it produces made by bundles mind, which need to decode, sort out, decipher, work out, make out, order, decrypt and scramble the info. To be suitable, we now have to be sure that gathering mind change regularly all with each other not to eat up all the imperativeness of the bundle head.

\section{Individual key:}

In this plan, each center has its own one of a kind key to scramble data. This key is simply known by the sink. Therefore, a message sent with this centre point goes around obscured on the framework right up until it accomplishes the drain. This course of activity is one of typically the better ways to oblige the application of the framework. Before extended, this course of activity confirms only correspondence in between a center and typically the sink.

\section{STEGANOGRAPHY \& RESULTS}

Anytime cryptography contains to produce jumbled a data, steganography is to hide the particular existence of information. By simply far the vast majority of example related to steganography can be uncovered along with data conceal within the substance, a image or even a video. At that will time these techniques are not able to become related to remote control transducer frameworks since information are missing tremendous. Whatever the case exhibit that will it is feasible to protect the information within the particular coating of the particular address 802 . 15. 4 display simply by using the upheaval through the sign for creating a steganography channel. This specific plan provides for safeguard the information, in any sort of situation in the event that an opponent knows the particular procedure, the specific data will be not a more guaranteed. E. Age One sports activity plan proposed by is to use an important age group. Each period or actually age, the sink transfers another step to the complete framework. This key will be utilized as verification for each and every middle point; to indicate outstanding spot with the platform. Just in case an unidentified middle endeavors to come in to the remote transducer orchestrate and if it does not get this key age, the particular framework will decay the mix. Another bit associated with leeway of the technique will be that it limits replacement attacks of a transducer as well as the remaking of the particular transducer to become reused inside the framework 


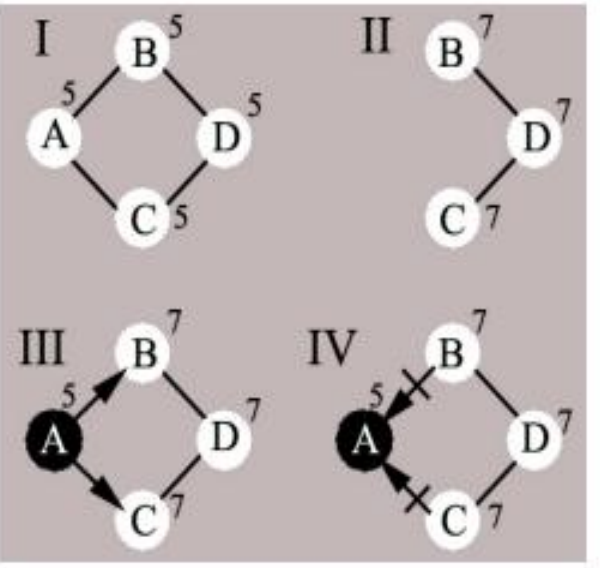

Fig 5.1 Steganography

An auto dvd unit is given within physique 5. 1, exactly where several transducers A, W, D, D are fairly regarding a transducer mastermind who else talks by using a symmetric couple insightful important center level. In Phase I, transducers have the key era 5. Within Stage 2, the center level is purged by a great demand, and during their nonappearance around the construction, the kitchen sink forward one more age group key 7 . Inside Phase III, the transducer The that has been reevaluated in addition to reinserted to the framework seems to make an growth request directly into the particular framework to transducer W and transducer D. Within Stage IV centre factors B and D deny the sales regarding a good, in light about the truth that going for the gander at their important age, they found really interesting. This tactic is normally imperativeness powerful and straightforward to deliver. In any circumstance this composed simply shut straight down frameworks, which cannot realize new center points. Within addition, you have the concern associated with any center point, which usually often cannot get the vital to propel time.

Fahrenheit. Confinement an instrument familiar to recognize malignant middle factors and especially wormhole ambushes is by by using a procedure with regard in order to finding geographically a center, due to the fact proposed. For this technique, the remote transducer system needs express transducers referred to as signal center point, which are transducers that realizing their very own territory position. For example helpful to them for the GPS NAVIGATION equipment. Making use of the limitation, in circumstance a transducer requesting inside order to join typically the framework, helps could possibly get these kinds of product sales and experiencing the alternative to examine its zone with their particular own hearing region. Guide concentrates will make a real mix section of their certain hearing region, and every single beacon center point, which often usually got the seeking with regard to admittance within the framework, may certainly cast a paquet with regard to a great area of the certain system that can listen closely to. The locale which often will get the finest quantity of ballots really need to be the certain place may be typically the new transducer. It shows an example of selection between four manage transducers A, W, C in addition to D. Earning a construction of their own website tuning in. These men and women vote for every single zone related with the system. They could evaluate the circumstance coming from the transducer which they will measure. The newest transducer should be seen inside most of the region with typically the many votes; within this specific point regarding reference typically the zone together with three or more casts a tally.

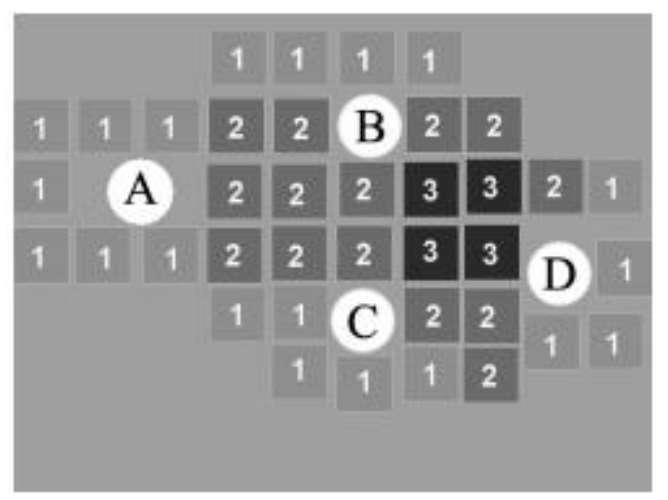

Fig 5.2 Confinement

In the event there is a wormhole attack combined with two destructive nodes, these are geo-located by simply beacon clients, which usually are going to be in a position to be too inside a position to determine the distance between generally the two nodes. They can see if this duration is higher than typically the conventional distance to get a relationship in a hop, in addition to and then discover the particular attack. Typically the issue associated with this fix is it requirements bright spot transducers outfitted with GPS UNIT gadget or recalibrated about the particular ground.

\section{G. Rely upon management}

One solution suggested by and also to use usually the mechanisms of believe in addition to status that may finish up becoming present in peer to observe networks community networks because well as market web sites as Amazon. In this particular kind of community such because wireless transducer sites, this is not easy, consequently from the huge number associated with nodes, to be capable to know which client can be very the malicious node. To find out plus protect the ethics associated with the network, each plus every node of the particular community monitors its nearby clients and their activities above time. Depending upon steps popular among the neighboring nodes, a client will increase an amount associated with trust of these systems, based on its status. Each time a node does not really carry out to ask for, its level of believe in falls if this client always sends properly information, its level of believe in increases.

By using these amounts of trust, a client will then select the best route for sending info. Instead of going by means of the speediest path (number of hops or territorial distance), the node may possibly opt to send their facts via nodes together with typically the highest stage of depend upon (the most dependable path). This type of mechanism is usually represented, wherever a computer A provides to send out data into a computer D. Instead regarding proceeding from the shortest approach which passes through Times, the

Blue Eyes Intelligence Engineering \& Sciences Publication 
industry node with a new level of trust regarding 3 (level is in between 0 and 10, 12 is the highest stage of trust) and probably a malicious node, typically the node A will send out information via nodes $\mathrm{M}$ and $\mathrm{C}$, which may have a new level of trust regarding 8 and 9 in addition to which propose the most dependable route. With this remedy, in addition, it uses a approach called watchdog. In typically the mechanism of watchdog, each and every communication between two clients A and B is usually heard by an more advanced node $\mathrm{C}$, found in typically the area of communication.

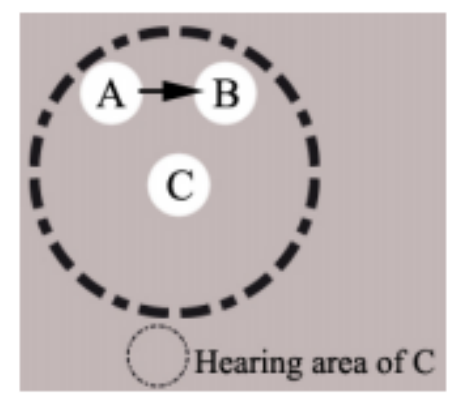

Fig 5.3 Route of Sending data

These methods make it possible to execute center points that are conceivably unsafe, and secure data to encounter these centers. Plans reliant on the trust the board are essentialness capable, and cannot use cryptography in a framework, which needn't waste time with a high security. Regardless, for frameworks that require most extraordinary security, they are not continually balanced. As needs be a harmful center that essentially record data on the framework, as in diminish opening attack, isn't generally perceivable.

\section{CONCLUSION}

Past due imaginative advances in remote control transducer frameworks have permitted sweeping utilization of this particular sort of framework. However, information increased 'til now susceptible against various attacks, which usually are often expressed in order to extraordinarily designated frameworks, or even particular to remote control transducer frameworks. A few programs of action are suggested by built up specialists to counter these ambushes, anyway as we demonstrated up in this post, they provide not a reply to all strikes. The particular low figuring power associated with transducers and especially their own obliged imperativeness are inconveniences to the association associated with front line methods, plus we are upwards 'til now filtering regarding methods of action, which often can support security, would certainly consolidate long term along together with a not too weak dormancy of transducers. Many of us have to recall of which they will be recycled really a new unique secure plan inside remote control transducer frames. The aspect of safety and also the disadvantage of a new remote transducers framework count after the application contact form that we get in order to be able to send.

\section{REFERENCES}

1. Kim S, Pakzad S, Culler DE, Demmel J, Fenves G, Glaser S, Turon M. Distant transducer frameworks for essential prosperity checking. SenSys, Campbell AT, Hat P, Heidemann JS (eds. ), ACM, 2006; 427-428.

2. Welsh M. Passing over a transducer orchestrate on a new working wellspring of liquid magma. USENIX Yearly Specific Meeting, General Monitor, USENIX, 2006.

3. Breads cook CR, Armijo E, Belka S, Benhabib Meters, Bhargava V, Burkhart And, Minassians Promotion, Dervisoglu Gary the tool man, Gutnik L, Haick MEGA BYTES, et al.. Remote transducer frameworks for home regenerative administrations AINA Workshops (2), IEEE PC Society, a few years ago; 832-837

4. Thierry AS, Francois SJ, sobreGentili Emmanuelle, Bernadette Chemical. Using remote control transducer create for crazy flame region. a under the radar event strategy of standard looking at device. Condition Personalities plus Mediterranean sea Region 06 ISEIMA '06 First globally Symposium upon, 2006

5. Malan D, Fulford-Jones $\mathrm{T}$, Welsh M, Moulton S Codeblue: An from the wristband transducer sort away framework for emergency restorative believed. Overall Workshop upon Wearable and Implantable Entire body Transducer Systems 2004

6. Carman DW, Krus PLAYSTATION, Matt BJ. Essentials plus techniques for spread transducer orchestrate security. Particular Report 00-010, NAI Labratories, System Companions, Inc., Glenwood, MD, 2k. [7] Akyildiz IF, Tu W, Sankarasubramaniam Y, Cayirci E. Handheld remote control transducer types out: the survey. Comput.Netw. 2002; 38(4): 393-422, doi: http://dx.doi.org/10.1016/S1389-1286(01)00302-4.

7. Al-Karaki JN, Kamal STRYGE. Coordinating methods in remote control transducer orchestrates: a study. IEEE Handheld remote control Comm., volume. 11, 2005; 628.

8. Crossbow development incorporation. $\mathrm{mpr} / \mathrm{mib}$ client's manual.

http://www.xbow.com/Backing/Support_pdf_files/MPR MIB/Series_Users_Manual.pdf 2010. [10] Zigbee organization. http://www.zigbee.org/2010.

9. Wooden A, Stankovic J. Repudiation of businesses in transducer frameworks. IEEE PC Oct 2002;

10. Parno B, Perrig A, Gligor VD. Distribute acknowledgment related to center stage replication assaults in transducer frameworks. IEEE Symposium upon Security plus Protection, IEEE PC Community, 2005; 49-63. [13] Wang X, Gu W, Schosek K, Chellappan S, Xuan D. Transducer straighten away plan under bodily assaults. ICCNMC, Address Information within Software building, volume. 3619, Lu X, Zhao Watts (eds. ), Springer, 2006; 23-32.

11. Hartung C, Balasalle M, Ryan R. Center stage manage transducer masterminds: The specific necessity for secure methods. Specific Report CUCS-988-04, Division society building, University associated with Colorado at Rock, 2005;

12. Karlof Chemical, Wagner D. Safe leading in remote transducer frames: strikes and countermeasures. Remarkably named Systems the 12 months 2003; 1(2-3): 293-315

13. SandeepSaurav Singh, Con. BevishJinila, "Transducer Node Failure Recognition making usage of Check Stage Recovery Algorithm", Fifth Worldwide conference on recent styles in Information Technology (ICRTIT), 8-9th April 2016, IEEE.

14. Sam Mathews, Y. BevishJinila (2014), "An effective strategy for pseudonym generation and changing scheme with privacy preservation for VANET", International 
Conference on Electronics and Communication Systems (ICECS) 2014, ISBN -978-1-4799-2321-2, pp. 1-6, IEEE

15. Y. BevishJinila, K. Komathy (2013), " A privacy preserving authentication framework for safety messages in vanet", 4th International Conference on Sustainable Energy and Intelligent System (SEISCON 2013), December 12-14, 2013, pp. 456-461, IET.

16. Sivasangari, A., Bhowal, S., Subhashini, R., Secure encryption in wireless body sensor networks, Advances in Intelligent Systems and Computing, 2019.

17. J.S.Vimali, Mekala Harinath Reddy, A Survey on Various Routing Protocols, International Conference on Human Computer Intreactions ICHCI'16. 